\title{
Ultralight Dark Matter Resonates with Binary Pulsars
}

\author{
Diego Blas, ${ }^{1, *}$ Diana López Nacir, ${ }^{1, \dagger}$ and Sergey Sibiryakov ${ }^{1,2,3, \$}$ \\ ${ }^{1}$ Theoretical Physics Department, CERN, CH-1211 Genève 23, Switzerland \\ ${ }^{2}$ Institute of Physics, LPPC, Ecole Polytechnique Fédérale de Lausanne, CH-1015 Lausanne, Switzerland \\ ${ }^{3}$ Institute for Nuclear Research of the Russian Academy of Sciences, 60th October Anniversary Prospect, 7a, 117312 Moscow, Russia
}

(Received 30 January 2017; published 29 June 2017)

\begin{abstract}
We consider the scenario where dark matter (DM) is represented by an ultralight classical scalar field performing coherent periodic oscillations. We point out that such DM perturbs the dynamics of binary systems either through its gravitational field or via direct coupling to ordinary matter. This perturbation gets resonantly amplified if the frequency of DM oscillations is close to a (half-)integer multiple of the orbital frequency of the system and leads to a secular variation of the orbital period. We suggest using binary pulsars as probes of this scenario and estimate their sensitivity. While the current accuracy of observations is not yet sufficient to probe the purely gravitational effect of DM, it already yields constraints on direct coupling that are competitive with other bounds. The sensitivity will increase with the upcoming radio observatories such as the Square Kilometer Array.
\end{abstract}

DOI: 10.1103/PhysRevLett.118.261102

Introduction.-Despite years of intensive research, the nature of dark matter (DM) remains unknown. An interesting possibility is that DM is represented by a very light boson with extremely weak, if any, coupling to the fields of the standard model; see Ref. [1] for a recent review. Such DM candidates are common in many models of new physics including the axion solution to the strong $C P$ problem [2-4], the relaxion mechanism for the origin of the electroweak symmetry breaking [5], and string theory [6,7]. Huge particle occupation numbers required to reproduce the DM density imply that such ultralight dark matter (ULDM) is well described by a classical scalar field $\Phi$. For a single field to be all of the DM, the anharmonicities of the potential are constrained to be small when the field starts oscillating at $H \sim m_{\Phi}$ (with $H$ the Hubble rate) [8]. Since the amplitude of the field at that moment is larger than its present amplitude in the galactic halo, we will neglect possible self-interactions of $\Phi$.

A lot of effort has been devoted to identify observations and experiments sensitive to ULDM. Very light candidates with masses $m_{\Phi} \lesssim 10^{-24} \mathrm{eV}$ are excluded as the dominant DM component by the observations of the cosmic microwave background and large-scale structure [9]; future observations are expected to push the lower bound to $m_{\Phi} \sim 10^{-23} \mathrm{eV} \mathrm{[10].}$ Slightly heavier ULDM in the range $m_{\Phi} \sim 10^{-23}-10^{-21} \mathrm{eV}$ can be probed by the Lyman- $\alpha$ forest, galaxy formation history, and the structure of galactic halos [11-16]; a complementary probe is provided by the Pulsar Timing

Published by the American Physical Society under the terms of the Creative Commons Attribution 4.0 International license. Further distribution of this work must maintain attribution to the author(s) and the published article's title, journal citation, and DOI.
Arrays (PTA) [17,18]. The mass range up to $m_{\Phi} \sim$ $10^{-18} \mathrm{eV}$ can, in principle, be accessible to $21 \mathrm{~cm}$ surveys [19]. Scalar fields with $m_{\Phi} \sim 10^{-14}-10^{-10} \mathrm{eV}$ would be produced by rotating stellar mass black holes via superradiance, which implies various observable signatures including gravitational wave emission in the LIGO sensitivity band [20,21]. Future studies of supermassive black holes can potentially access lighter masses $m_{\Phi} \sim 10^{-20}-10^{-15} \mathrm{eV}$. The gravitational effect of ULDM on laser interferometers was explored in Ref. [22].

The previous observations probe purely gravitational interactions of ULDM. If ULDM has a direct coupling to ordinary matter the possibilities to test it are more diverse and depend on specific models. A rather generic effect of ULDM is periodic modulation of the standard model couplings and particle masses with time. A number of proposals have been recently put forward to search for such variations using atomic clocks [8,23-27], accelerometers [28], resonant-mass detectors [29], laser and atom interferometry [30-33].

In this work we propose using observations of binary pulsars as a probe of ULDM in the mass range $m_{\Phi} \sim$ $10^{-23}-10^{-18} \mathrm{eV}$. The exquisite precision of the measurements combined with the clean theoretical description makes binary pulsars highly sensitive to new physics that affects the dynamics of massive objects [34,35]. This property has already been exploited to constrain alternatives to general relativity [36,37] and led to the suggestion to use binary pulsars as resonant detectors of the stochastic gravitational wave (GW) background [38] (see Refs. [39-43] for related earlier works). The influence of DM composed of weakly interacting heavy particles on the dynamics of binary pulsars was studied in Ref. [44].

The main idea of our approach is close in spirit to Refs. [38-43] and can be summarized as follows. The 
ULDM field $\Phi$ in the galactic halo represents a collection of plane waves with frequencies (we use units $c=\hbar=1$.) $\omega_{\Phi} \simeq m_{\Phi}+m_{\Phi} v^{2} / 2$ and momenta $k_{\Phi} \simeq m_{\Phi} v$, where $v \sim 10^{-3}$ is the typical virial velocity in the halo. Neglecting the term $m_{\Phi} v^{2} / 2$ in the frequency we obtain the general form of the ULDM field,

$$
\Phi(\mathbf{x}, t)=\Phi_{0}(\mathbf{x}) \cos \left(m_{\Phi} t+\Upsilon(\mathbf{x})\right),
$$

where $\Phi_{0}(\mathbf{x})$ and $\Upsilon(\mathbf{x})$ are slowly varying functions of position. A binary system embedded in the DM background (1) will experience periodic perturbation due to the change in the gravitational field of $\Phi$ and, in the presence of a direct coupling, due to the change in the masses of the stars in the binary. If the frequency of the perturbation happens to be close to an integer multiple of the binary orbital frequency, its effect is resonantly amplified and leads to a secular change in the orbital period that can be searched for experimentally. We now proceed to the quantitative discussion. We start with the case when DM and ordinary matter interact only gravitationally.

ULDM interacting only through gravity.-The energymomentum of a free massive oscillating field (1) corresponds to the density and pressure [17],

$\rho_{\mathrm{DM}}=\frac{m_{\Phi}^{2} \Phi_{0}^{2}}{2}, \quad p_{\mathrm{DM}}=-\rho_{\mathrm{DM}} \cos \left(2 m_{\Phi} t+2 \Upsilon\right)$.

The latter generates an oscillating perturbation of the metric. To find this we use the Newtonian gauge,

$$
d s^{2}=-(1+2 \phi) d t^{2}+(1-2 \psi) \delta_{i j} d x^{i} d x^{j},
$$

and write down the trace of the $(i j)$ Einstein equations,

$$
6 \ddot{\psi}+2 \Delta(\phi-\psi)=24 \pi G p_{\mathrm{DM}} .
$$

Neglecting the spatial gradients and using Eq. (2) we obtain,

$$
\ddot{\psi}=-4 \pi G \rho_{\mathrm{DM}} \cos \left(2 m_{\Phi} t+2 \Upsilon\right) .
$$

This can be viewed as a standing scalar GW. Similarly to the usual GW's, it produces an extra relative acceleration between the bodies in a binary system. This is conveniently written in the Fermi normal coordinates associated with the center of mass of the binary [41],

$$
\delta \ddot{r}^{i}=-\delta R_{0 j 0}^{i} r^{j}=-\ddot{\psi} r r^{i},
$$

where $r^{i}$ is the vector connecting the two bodies and $\delta R_{0 j 0}^{i}$ is the contribution of GW into the corresponding components of the Riemann tensor. In the last equality we evaluated $\delta R_{0 j 0}^{i}$ in the conformal gauge (3) since it is coordinate independent at the linearized level.

Next, we compute the change in the energy of a binary system with masses $M_{1,2}$ during one orbital period $P_{b}$ due to its interaction with ULDM,

$$
\begin{aligned}
\delta E_{b} & =\mu \int_{0}^{P_{b}} \dot{r}^{i} \delta \ddot{r}^{i} d t \\
& =4 \pi G \rho_{\mathrm{DM}} \mu \int_{0}^{P_{b}} \dot{r}(t) r(t) \cos \left(2 m_{\Phi} t+2 \Upsilon\right) d t,
\end{aligned}
$$

where $r$ is the distance between the bodies and $\mu \equiv$ $M_{1} M_{2} / M_{1}+M_{2}$ is the reduced mass of the system. The energy exchange is most efficient when the orbital period is close to an integer multiple of the period of metric oscillations. Given that $P_{b} \propto\left|E_{b}\right|^{-3 / 2}$, the change in Keplerian energy leads to a secular drift of the orbital period. Defining

$$
\delta \omega=2 m_{\Phi}-2 \pi N / P_{b}, \quad|\delta \omega| \ll 2 m_{\Phi},
$$

and using the standard formulas of Keplerian mechanics, we obtain the time derivative of the period averaged over time intervals $\Delta t$ satisfying $P_{b} \ll \Delta t \ll 2 \pi / \delta \omega$,

$$
\begin{aligned}
\left\langle\dot{P}_{b}\right\rangle & =-6 G \rho_{\mathrm{DM}} P_{b}^{2} \frac{J_{N}(N e)}{N} f(t) \\
& \simeq-1.6 \times 10^{-17}\left(\frac{\rho_{\mathrm{DM}}}{0.3 \frac{\mathrm{GeV}}{\mathrm{cm}^{3}}}\right)\left(\frac{P_{b}}{100 \mathrm{~d}}\right)^{2} \frac{J_{N}(N e)}{N} f(t),
\end{aligned}
$$

where

$$
f(t)=\sin \left(\delta \omega t+2 m_{\Phi} t_{0}+2 \Upsilon\right),
$$

$J_{N}(x)$ are Bessel functions, $e$ is the orbital eccentricity, and $t_{0}$ is the time of the first periastron passage since $t=0$. In the second line of Eq. (7) we have normalized $\rho_{\mathrm{DM}}$ to the local DM density $\sim 0.3 \mathrm{GeV} / \mathrm{cm}^{3}$ in the neighborhood of the Solar System. We observe that, depending on the relative phase between the orbital motion and the ULDM oscillations, the sign of $\left\langle\dot{P}_{b}\right\rangle$ can be negative (decrease of the binary system energy) or positive (increase of the energy). Furthermore, the sign alternates in time with the period $2 \pi / \delta \omega \gg P_{b}$, which can be used to discriminate this effect from other contributions to the measured $\dot{P}_{b}$, such as, e.g., those due to the acceleration of the binary with respect to the Solar System.

The expression (7) implies that the effect vanishes for circular orbits $(e=0)$ and grows with the orbital eccentricity. Besides, it is stronger for systems with large orbital periods. These points are illustrated in Fig. 1. We see that slow nonrelativistic systems with orbital periods of tens to hundreds of days and high eccentricity present suitable targets to search for ULDM in the mass range $m_{\Phi}=10^{-23}-10^{-21} \mathrm{eV}$. At present there is a dozen of known binary pulsars satisfying these requirements [45]; this number is expected to increase dramatically with the operation of the Square Kilometer Array [46]. Note that for such systems the strength of the resonance on higher harmonics $(N \geq 2)$ is comparable to the strength of the 


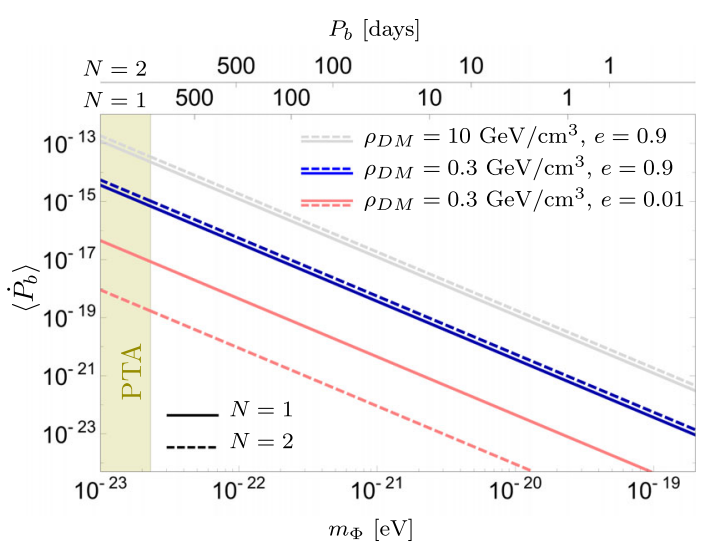

FIG. 1. Secular derivative of the orbital period given in Eq. (7) as a function of the dark matter mass. We have set $f(t)=-1$ for the numerical estimate. Solid lines assume resonances for $N=1$ $\left(m_{\Phi}=\pi / P_{b}\right)$, while dashed ones are for $N=2\left(m_{\Phi}=2 \pi / P_{b}\right)$. The corresponding orbital periods are shown on the two top axes. The pink (lower) lines correspond to $\rho_{\mathrm{DM}}=0.3 \mathrm{GeV} / \mathrm{cm}^{3}$ and $e=0.01$, the blue (middle) lines are for the same $\rho_{\mathrm{DM}}$ but $e=0.9$, while the gray (upper) lines correspond to $\rho_{\mathrm{DM}}=$ $10 \mathrm{GeV} / \mathrm{cm}^{3}$ and $e=0.9$. The olive band on the left marks the regions $m_{\Phi} \lesssim 2.3 \times 10^{-23} \mathrm{eV}$, which can be probed by future pulsar timing arrays [17].

main resonance $(N=1)$, which implies that a single eccentric binary probes several different ULDM masses.

Detecting $\left\langle\dot{P}_{b}\right\rangle$ induced by pure gravitational interaction of ULDM will be challenging. It will require the accuracy of at least $10^{-16}$ in the determination of this quantity for nonrelativistic binaries. Currently such precision has been achieved for the double pulsar PSR J0737-3039A/B [47], whose orbital period is, however, too short $\left(P_{b} \approx 0.1 \mathrm{~d}\right)$ to be sensitive to the gravitational effect of ULDM (the situation is different in the presence of direct coupling, see below). One may hope that the peculiar periodic modulation of $\left\langle\dot{P}_{b}\right\rangle$ predicted by Eq. (7) and the expected correlation of its phase among systems located within the coherence length of ULDM can be used to increase the sensitivity of the search. A proper estimation of the measurability of these effects is beyond the purpose of this Letter. Still, let us comment on what could be an optimal scenario. For the modulation to be, in principle, detectable one needs to observe the system for at least half of the period $T_{\text {obs }} \gtrsim T_{\text {mod }} / 2=\pi / \delta \omega$. The uncertainty in the measurement of $\left\langle\dot{P}_{b}\right\rangle$ for a constant secular drift scales as $T_{\mathrm{obs}}^{-5 / 2}$ [48]. For a modulated signal, the drift in $P_{b}$ can be considered as approximately constant for half of the period. So, we will take as a conservative estimate for the error $\Delta\left\langle\dot{P}_{b}\right\rangle \propto\left(T_{\text {mod }} / 2\right)^{-5 / 2}$. Thus, long modulations are preferable for detection (the closer the system is to the resonance, the better); still, they should stay within the range $T_{\text {mod }} \lesssim 2 T_{\text {obs }}$. An additional handle is provided by ULDM-induced secular variations in other orbital parameters [49].
A complementary way to improve the sensitivity to ULDM is to look for binary systems in a denser DM environment. The Navarro-Frenk-White halo profile [50] predicts an increase of $\rho_{\text {DM }}$ up to $\sim 10 \mathrm{GeV} / \mathrm{cm}^{3}$ within $0.5 \mathrm{kpc}$ distance from the Galactic center (In the ULDM scenario the inner part of the Milky Way halo can contain a solitonic core which may further increase $\rho_{\mathrm{DM}}$ in the vicinity of the Galactic center [51]. However, the size of the core is smaller than $0.5 \mathrm{kpc}$.). The impact of such increase on $\left\langle\dot{P}_{b}\right\rangle$ is shown in Fig. 1 by gray lines.

The possibility to discover the previous effects in future measurements strongly depends on the characteristics of each individual observed system. Since these are currently unknown, a precise forecast of detectability is impossible at the present stage. An insight can be gained from the analysis of simulated mock samples. This study must be performed in the future to assess the actual measurability of the purely gravitational effect.

ULDM directly coupled to matter.-We now assume that ULDM interacts directly with the bodies in the binary by affecting their masses,

$$
M_{1,2}(\Phi)=M_{1,2}(1+\alpha(\Phi)), \quad|\alpha(\Phi)| \ll 1 .
$$

For simplicity, we focus on the case of universal coupling (Namely, we assume all particle species couple to the same effective metric that depends on the scalar field and hence the weak equivalence principle is preserved.), the case of different couplings will be treated elsewhere [49]. In what follows we will neglect the gravitational interaction between ULDM and the binary. Then in the nonrelativistic limit the system is described by the Lagrangian,

$L=M_{1}(\Phi)\left(1+\frac{v_{1}^{2}}{2}\right)+M_{2}(\Phi)\left(1+\frac{v_{2}^{2}}{2}\right)+\frac{G M_{1}(\Phi) M_{2}(\Phi)}{r}$.

By combining the equations of motion of the two bodies we obtain that their relative acceleration acquires a contribution proportional to the direct coupling,

$$
\delta \ddot{r}^{i}=-\frac{d \alpha}{d \Phi} \dot{\Phi} \dot{r}^{i}-\alpha(\Phi) \frac{G\left(M_{1}+M_{2}\right) r^{i}}{r^{3}} .
$$

As in the case of the pure gravitational interaction, this leads to the change in the Keplerian energy and hence a secular drift of the orbital period. Below we consider two choices for the function $\alpha(\Phi)$.

Linear coupling $\alpha(\Phi)=\Phi / \Lambda_{1}$. - In this case the condition for the resonance reads,

$$
\delta \omega=m_{\Phi}-2 \pi N / P_{b}, \quad|\delta \omega| \ll m_{\Phi} .
$$

Evaluating the energy change due to Eq. (9) and relating it to the derivative of the orbital period we obtain, 


$$
\begin{aligned}
\left\langle\dot{P}_{b}\right\rangle \simeq & 2.5 \times 10^{-12}\left(\frac{\rho_{\mathrm{DM}}}{0.3 \frac{\mathrm{GeV}}{\mathrm{cm}^{3}}}\right)^{\frac{1}{2}}\left(\frac{P_{b}}{100 \mathrm{~d}}\right) \\
& \times\left(\frac{10^{23} \mathrm{GeV}}{\Lambda_{1}}\right) J_{N}(N e) \sin \left(\delta \omega t+m_{\Phi} t_{0}+\Upsilon\right) .
\end{aligned}
$$

Quadratic coupling $\alpha(\Phi)=\Phi^{2} /\left(2 \Lambda_{2}^{2}\right)$. - Here we are back to the resonant condition (6) and the ULDM-induced variation of the orbital period is

$$
\begin{aligned}
\left\langle\dot{P}_{b}\right\rangle \simeq & 1.1 \times 10^{-11}\left(\frac{\rho_{\mathrm{DM}}}{0.3 \frac{\mathrm{GeV}}{\mathrm{cm}^{3}}}\right)\left(\frac{P_{b}}{100 \mathrm{~d}}\right)^{2} \\
& \times\left(\frac{10^{16} \mathrm{GeV}}{\Lambda_{2}}\right)^{2} \frac{J_{N}(N e)}{N} \sin \left(\delta \omega t+2 m_{\Phi} t_{0}+2 \Upsilon\right) .
\end{aligned}
$$

Similar to Eq. (7), the expressions (10) and (11) vanish for circular orbits implying that systems with higher eccentricity are preferred to search for the effect.

Current constraints on $\Lambda_{1,2}$ come from several sources. Linear coupling to a light scalar field modifies the attraction between massive bodies. This occurs even if $\Phi$ is not the DM and has been constrained by Doppler tracking of the Cassini spacecraft [52] yielding $\Lambda_{1} \gtrsim 10^{21} \mathrm{GeV}$. On the other hand, this bound does not apply to the quadratic coupling, leaving a much milder constraint from astrophysical processes and short-distance tests of gravity $\Lambda_{2} \gtrsim$ $10^{4} \mathrm{GeV}$ [23,53]. For a scalar field comprising ULDM additional bounds arise due to the constraints on the GW background. Indeed, the direct coupling of the scalar field to masses (8) can be absorbed by a redefinition of the metric to the so-called Jordan frame

$$
g_{\mu \nu} \mapsto \bar{g}_{\mu \nu}=g_{\mu \nu}(1+2 \alpha(\Phi)) .
$$

Test bodies move along geodesics of $\bar{g}_{\mu \nu}$ which has an oscillatory component due to oscillations of $\Phi$-this is a scalar GW. The amplitude of such oscillations has been constrained by PTA data [18] in the low frequency region $\left(f<10^{-7} \mathrm{~Hz}\right)$ and by Cassini tracking (Ref. [54] presents the limits on the amplitude $h_{c}$ of the stochastic background of transverse GW's that can be translated into a bound on the scalar GW amplitude $\psi_{c}$ by identifying $h_{c} \simeq \sqrt{15} \psi_{c}$. This is slightly different from the identification $h_{c} \simeq$ $2 \sqrt{3} \psi_{c}$ used in Refs $[17,18]$ because, unlike pulsar timing, the light time between the spacecraft and Earth is less than the GW period, which leads to a different expression for the average of the stochastic signal.) [54] in the frequency range $f=10^{-6}-10^{-3} \mathrm{~Hz}$.

Limits on an ULDM-induced contribution into $\dot{P}_{b}$ in the timing model of binary systems can be used to put further bounds on the couplings $\Lambda_{1,2}^{-1}$. Taking the reported error in the determination of intrinsic $\dot{P}_{b}$ for several known systems as an upper limit on the ULDM-induced contribution we obtain the constraints presented in Figs. 2, 3. In deriving them we have set the oscillating factors in Eqs. (10), (11) to one for the sake of the argument. One observes that they are competitive with the existing bounds. In particular, the Hulse-Taylor pulsar B1913+16 [55] provides the most sensitive probe of the direct ULDM coupling for $m_{\Phi}$ satisfying the appropriate resonance condition; whereas the systems J1903+0327 [56] and J1748-2021B [57] give the strongest constraints on the quadratic coupling $\Lambda_{2}^{-1}$ in the range of $m_{\Phi}$ from $2 \times 10^{-22}$ to $2 \times 10^{-21} \mathrm{eV}$. The situation will further improve with the increase of precision in binary pulsar timing and discovery of new binary systems. To illustrate this, we report the bounds one would obtain assuming that the systems considered before are timed at the best current precision (orange symbols in Figs. 2 and 3). Realistically, this precision may not be achievable for some of the systems presented in the plots due to various sources of intrinsic uncertainty in determination of $\dot{P}_{b}$. Nevertheless, it is reasonable to expect that future surveys will discover new binary pulsars suitable for precision timing and having periods and eccentricity similar to those of already existing systems. Note that valuable constraints on ULDM with masses $m_{\Phi} \sim 10^{-19}-10^{-18} \mathrm{eV}$ can be obtained even from

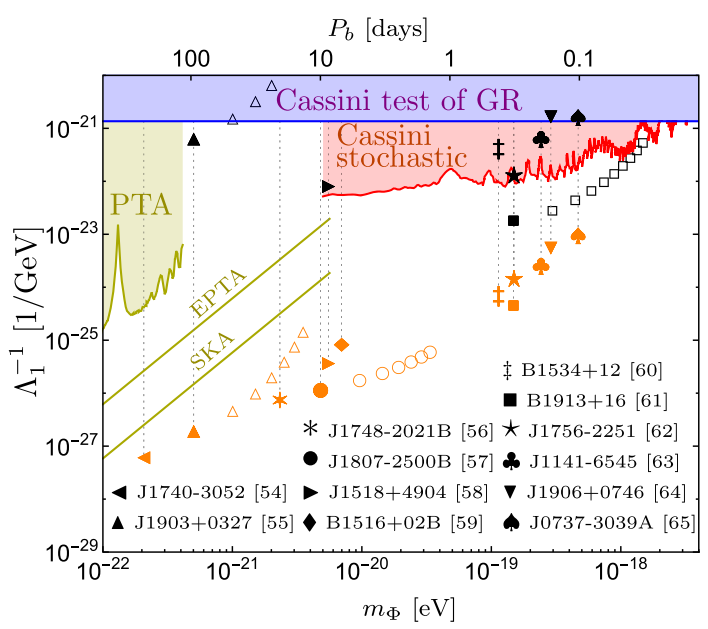

FIG. 2. Sensitivity of binary pulsar observations to the linear coupling $\Lambda_{1}^{-1}$ between ULDM and ordinary matter for several known systems (see the indicated references for their description). Black symbols are constraints derived using the existing data on $\dot{P}_{b}$; values above the symbols are excluded. Orange symbols show the sensitivity that can be achieved assuming $\dot{P}_{b}$ is measured for a given system with the accuracy $10^{-16}$ (see comments on the feasibility of such measurements in the main text). Empty symbols correspond to resonances on higher harmonics $(N \geq 2)$. The colored regions of the ULDM parameter space are excluded by PTA [18] (olive), the Cassini test of general relativity [52] (violet), and the Cassini bound on stochastic GW background [54] (red). Olive lines show future sensitivities of the European Pulsar Timing Array (upper) and the Square Kilometer Array (lower) as estimated in Ref. [28]. 


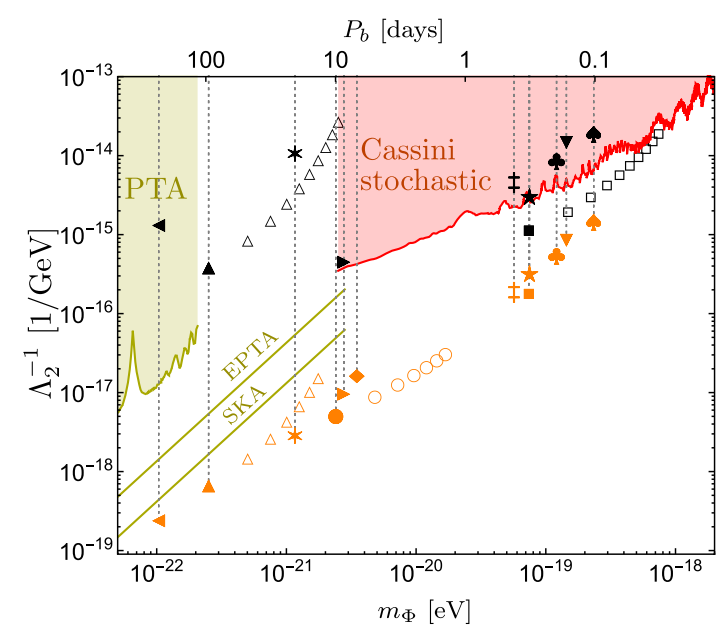

FIG. 3. Same as Fig. 2, but for the case of quadratically coupled ULDM. There are no constraints on $\Lambda_{2}^{-1}$ from Solar System tests of general relativity.

fast binaries with periods down to a few hours. In the lowmass region binary pulsar observations can be complementary to future PTA.

The precise values of the bounds from Figs. 2, 3 should be taken with caution. First, we have set the sine factors in Eqs. (10), (11) to 1, whereas their accidental suppression if the phase happens to be close to an integer multiple of $\pi$ is not excluded. This option would be reliably ruled out by studying an ensemble of systems, which would allow us to average over the phases. At the moment such a study is impossible due to the lack of statistics. Second, in the case of quadratic coupling one should take into account the screening effect when relating the bounds on $\Lambda_{2}$ from binary pulsars to the parameters in the particle physics Lagrangian. Indeed, a quadratically coupled scalar field acquires effective mass $m_{\text {eff }}^{2} \sim \rho / \Lambda_{2}^{2}$ inside an object with density $\rho$. If the corresponding Compton wavelength is shorter than the size of the object, the field inside the object gets frozen at $\Phi=0$ and only an outer layer of width $m_{\text {eff }}^{-1}$ interacts with ULDM. This can degrade the bounds on $\Lambda_{2}^{-1}$ by a few orders of magnitude compared to those shown on Fig. 3 [49].

A peculiarity of the binary pulsar constraint is that every single system is sensitive to ULDM masses only in a few narrow bands corresponding to resonances on the first $N \lesssim$ 10 harmonics. Conservatively, one can require that the system stays in resonance during the whole observational campaign, so that the changes in $P_{b}$ induced by ULDM accumulate over the time; this would maximize the sensitivity of observations to the effect. This yields an estimate of the band width $\delta \omega \sim 5 \times 10^{-23} \mathrm{eV} /$ (years of observation). While this is much smaller than the total mass range of interest, the $O\left(10^{3}\right)$ binary systems with different periods expected to be discovered by SKA [46] will allow for significant coverage.
We thank Vitor Cardoso, Emmanuel Fonseca, Paulo Freire, Gian Giudice, Michelangelo Mangano, David Pirtskhalava, and Valery Rubakov for discussions. The work of S. S. is supported by the Swiss National Science Foundation.

*Diego.Blas@cern.ch

†iana.Laura.Lopez.Nacir@cern.ch

*Sergey.Sibiryakov@cern.ch

[1] D. J. E. Marsh, Phys. Rep. 643, 1 (2016)

[2] R. D. Peccei and H. R. Quinn, Phys. Rev. Lett. 38, 1440 (1977).

[3] F. Wilczek, Phys. Rev. Lett. 40, 279 (1978).

[4] S. Weinberg, Phys. Rev. Lett. 40, 223 (1978).

[5] P. W. Graham, D. E. Kaplan, and S. Rajendran, Phys. Rev. Lett. 115, 221801 (2015).

[6] P. Svrcek and E. Witten, J. High Energy Phys. 06 (2006) 051.

[7] A. Arvanitaki, S. Dimopoulos, S. Dubovsky, N. Kaloper, and J. March-Russell, Phys. Rev. D 81, 123530 (2010).

[8] A. Arvanitaki, J. Huang, and K. Van Tilburg, Phys. Rev. D 91, 015015 (2015).

[9] R. Hložek, D. Grin, D. J. E. Marsh, and P. G. Ferreira, Phys. Rev. D 91, 103512 (2015).

[10] R. Hložek, D. J. E. Marsh, D. Grin, R. Allison, J. Dunkley, and E. Calabrese, Phys. Rev. D 95, 123511 (2017).

[11] W. Hu, R. Barkana, and A. Gruzinov, Phys. Rev. Lett. 85, 1158 (2000).

[12] L. Amendola and R. Barbieri, Phys. Lett. B 642, 192 (2006).

[13] B. Bozek, D. J. E. Marsh, J. Silk, and R. F. G. Wyse, Mon. Not. R. Astron. Soc. 450, 209 (2015).

[14] H.-Y. Schive, T. Chiueh, T. Broadhurst, and K.-W. Huang, Astrophys. J. 818, 89 (2016).

[15] A. Sarkar, R. Mondal, S. Das, S. Sethi, S. Bharadwaj, and D. J. E. Marsh, J. Cosmol. Astropart. Phys. 04 (2016) 012.

[16] L. Hui, J. P. Ostriker, S. Tremaine, and E. Witten, Phys. Rev. D 95, 043541 (2017).

[17] A. Khmelnitsky and V. Rubakov, J. Cosmol. Astropart. Phys. 02 (2014) 019.

[18] N. K. Porayko and K. A. Postnov, Phys. Rev. D 90, 062008 (2014).

[19] D. J. E. Marsh, Phys. Rev. D 91, 123520 (2015).

[20] A. Arvanitaki and S. Dubovsky, Phys. Rev. D 83, 044026 (2011).

[21] A. Arvanitaki, M. Baryakhtar, S. Dimopoulos, S. Dubovsky, and R. Lasenby, Phys. Rev. D 95, 043001 (2017).

[22] A. Aoki and J. Soda, Int. J. Mod. Phys. D 26, 1750063 (2017).

[23] A. Derevianko and M. Pospelov, Nat. Phys. 10, 933 (2014).

[24] K. Van Tilburg, N. Leefer, L. Bougas, and D. Budker, Phys. Rev. Lett. 115, 011802 (2015).

[25] Y. V. Stadnik and V. V. Flambaum, Phys. Rev. Lett. 115, 201301 (2015).

[26] A. Hees, J. Guéna, M. Abgrall, S. Bize, and P. Wolf, Phys. Rev. Lett. 117, 061301 (2016).

[27] Y. V. Stadnik and V. V. Flambaum, Phys. Rev. A 94, 022111 (2016). 
[28] P. W. Graham, D. E. Kaplan, J. Mardon, S. Rajendran, and W. A. Terrano, Phys. Rev. D 93, 075029 (2016).

[29] A. Arvanitaki, S. Dimopoulos, and K. Van Tilburg, Phys. Rev. Lett. 116, 031102 (2016).

[30] Y. V. Stadnik and V. V. Flambaum, Phys. Rev. Lett. 114, 161301 (2015).

[31] Y. V. Stadnik and V. V. Flambaum, Phys. Rev. A 93, 063630 (2016).

[32] A. A. Geraci and A. Derevianko, Phys. Rev. Lett. 117, 261301 (2016).

[33] A. Arvanitaki, P. W. Graham, J. M. Hogan, S. Rajendran, and K. Van Tilburg, arXiv:1606.04541.

[34] R. N. Manchester, Int. J. Mod. Phys. D 24, 1530018 (2015).

[35] M. Kramer, Int. J. Mod. Phys. D 25, 1630029 (2016).

[36] N. Wex, arXiv:1402.5594.

[37] C. M. Will, Living Rev. Relativ. 17, 4 (2014).

[38] L. Hui, S. T. McWilliams, and I.-Sheng. Yang, Phys. Rev. D 87, 084009 (2013).

[39] B. Bertotti, Astrophys. Lett. 14, 51 (1973).

[40] V. N. Rudenko, Sov. Astron. 19, 270 (1975).

[41] B. Mashhoon, Astrophys. J. 223, 285 (1978).

[42] M. S. Turner, Astrophys. J. 233, 685 (1979).

[43] B. Mashhoon, B. J. Carr, and B. L. Hu, Astrophys. J. 246, 569 (1981).

[44] P. Pani, Phys. Rev. D 92, 123530 (2015).
[45] http://www.atnf.csiro.au/people/pulsar/psrcat/; R. N. Manchester, G. B. Hobbs, A. Teoh, and M. Hobbs, Astron. J. 129, 1993 (2005).

[46] M. Kramer and B. Stappers, arXiv:1507.04423.

[47] M. S. Kehl, N. Wex, M. Kramer, and K. Liu, arXiv: 1605.00408

[48] T. Damour and J. H. Taylor, Phys. Rev. D 45, 1840 (1992).

[49] D. Blas, D. L. Nacir, and S. Sibiryakov (to be published).

[50] J. F. Navarro, C. S. Frenk, and S. D. M. White, Astrophys. J. 462, 563 (1996).

[51] H.-Y. Schive, T. Chiueh, and T. Broadhurst, Nat. Phys. 10, 496 (2014).

[52] B. Bertotti, L. Iess, and P. Tortora, Nature (London) 425, 374 (2003).

[53] K. A. Olive and M. Pospelov, Phys. Rev. D 77, 043524 (2008).

[54] J. W. Armstrong, L. Iess, P. Tortora, and B. Bertotti, Astrophys. J. 599, 806 (2003).

[55] J. M. Weisberg and Y. Huang, Astrophys. J. 829, 55 (2016).

[56] P. C. C. Freire et al., Mon. Not. R. Astron. Soc. 412, 2763 (2011).

[57] P. C. C. Freire, S. M. Ransom, S. Begin, I. H. Stairs, J. W. T. Hessels, L. H. Frey, and F. Camilo, Astrophys. J. 675, 670 (2008). 